\title{
Instability of a fluid-fluid interface in driven colloidal mixtures
}

\author{
A Wysocki and H Löwen \\ Institut für Theoretische Physik II, Heinrich-Heine-Universität Düsseldorf, Universitätsstraße 1, \\ D-40225 Düsseldorf, Germany \\ E-mail: adam@thphy.uni-duesseldorf.de and hlowen@thphy.uni-duesseldorf.de
}

Received 23 July 2004, in final form 14 September 2004

Published 1 October 2004

Online at stacks.iop.org/JPhysCM/16/7209

doi:10.1088/0953-8984/16/41/004

\begin{abstract}
A Rayleigh-Taylor-like interface instability is studied in a compressible Brownian Yukawa fluid mixture on the 'molecular' scales of length and time of the individual particles. As a model, a two-dimensional phase-separated symmetric binary mixture of colloidal particles of type A and B with a fluidfluid interface separating an A-rich phase from a B-rich phase is investigated, by means of Brownian computer simulations, when brought into non-equilibrium via a constant external driving field which acts differently on the different particles and perpendicular to the interface. Two different scenarios are observed which occur either for high or for low interfacial free energies as compared to the driving force. In the first scenario for high interfacial tension, the critical wavelength $\lambda_{\mathrm{c}}$ of the unstable interface modes is in good agreement with the classical Rayleigh-Taylor formula provided that dynamically rescaled values for the interfacial tension are used. The wavelength $\lambda_{c}$ increases with time, representing an effect of self-healing of the interface due to a local density increase near the interface. The Rayleigh-Taylor formula is confirmed even if $\lambda_{\mathrm{c}}$ is of the order of a molecular correlation length. In the second scenario for very large driving forces as compared to the interfacial line tensions, on the other hand, the particles penetrate the interface easily due to the driving field and form microscopic lanes with a width different from the predictions of the classical Rayleigh-Taylor formula. The results are of relevance for phase-separating colloidal mixtures in a gravitational or electric field.
\end{abstract}

\section{Introduction}

Interface instabilities in driven non-equilibrium systems are well known from macroscopic hydrodynamics [1, 2]. Examples include the classic Rayleigh-Taylor instability [1] of a heavy liquid on top of another lighter liquid, the Mullins-Sekerka fingering instability [3, 4] in diffusive systems and the Saffmann-Taylor instability [5, 6] for compressed liquids of 
different viscosities. However, what is much less clear is the microscopic origin of these instabilities, i.e. a view which resolves the microscopic discrete particle trajectories causing the instability. Recently there has been progress in simulating large systems with 10000 10000000 discrete particles forming an interface [7-10]. In particular, the microscopic origin of the Rayleigh-Taylor instability was explored in huge molecular dynamics [7, 9, 10] and direct numerical [8] computer simulations. A natural question concerns the applicability of coarse-grained hydrodynamics to microscopic spatial dimensions. The basic quantities entering in hydrodynamics such as the viscosity and the surface tension are quantities which are only defined for large systems and exhibit finite size corrections when applied to small inhomogeneities.

Another approach to interfacial instabilities is via mesoscopic colloidal suspensions which introduce the fascinating possibility of studying the particle trajectories in real space by means of video microscopy, in quasi-two-dimensional suspensions [11]. Well-characterized colloidal suspensions serve as model systems in which to address many questions of manybody systems [12,13] including interfaces [14-19]. The strength of an external field is easily tunable for colloids in striking contrast to the case for molecular liquids. The dynamics of the mesoscopic colloidal particles which are embedded in a microscopic solvent is, however, Brownian rather than Newtonian [20]—such that dynamical quantities are different. To the best of our knowledge, for Brownian fluids nothing is known about the onset of the RayleighTaylor instability on a length scale of interparticle distances. Clearly, it will be qualitatively different from the molecular dynamics case where heat will be generated and inertia effects can lead to turbulence $[7,9,10]$. Mixtures of colloids and polymers, with a real-space analysis of the colloid trajectories, represent a valuable system in which to look at interfacial instabilities for Brownian particles in external driving fields [14].

In the present paper we study, by means of Brownian dynamics computer simulations, the particle-resolved onset of the Rayleigh-Taylor instability for Brownian fluids. In our model, an interface separating an A-rich from an A-poor fluid is exposed to an external field which acts differently on the different particles and is directed perpendicular to the interface. In order to keep the model simple [23] and to link to two-dimensional colloidal suspensions, we take two spatial dimensions and a symmetric equimolar mixture interacting via a Yukawa pair potential. Most of our characteristics, however, will carry over to three-dimensional systems, to asymmetric mixtures and to different interparticle interactions.

The external field is so large that it will induce local density inhomogeneities such that the fluid is compressible. We observe two different scenarios which occur either for high or for low interfacial free energies as compared to a typical thermal energy per average particle distance. In the first scenario for high interfacial tension, an interfacial instability is observed which is driven by the competition of the cost in interfacial tension versus gain in potential energy, similar in spirit to the classical Rayleigh-Taylor instability. The classical threshold value for the wavelength of unstable interface perturbations is confirmed provided that the line tension is dynamically rescaled in terms of the actual local density at the interface. The critical wavelength separating stable from unstable density undulations increases with time. This can be interpreted as a 'self-healing effect' for the interface, caused by a local density increase near the interface. In the second scenario for small interfacial tensions, on the other hand, the particles easily penetrate the interface due to the driving field and form microscopic lanes, similarly to in previous simulation studies [24-28]. In this case the classical threshold for the unstable wavelength is smaller than a molecular correlation length, such that a breakdown of macroscale hydrodynamics is expected. Our results are of relevance for phase-separating colloidal mixtures in a gravitational or electric field where similar effects have recently been reported by Aarts and co-workers [14] and for settling granular grains [21, 22]. 
The paper is organized as follows. In section 2, we define the model used and describe briefly our simulation technique. As a prerequisite, a microscopic calculation of the density profile and the line tension is presented in section 3. We further review the classical RayleighTaylor instability briefly in section 4 . Results for the interface instability for high line tensions are presented in section 5, while the case of small line tensions is described in section 6 . Conclusions are given in section 7. In particular, we discuss some possible experimental tests.

\section{The model}

In our model [25], we consider a symmetric binary colloidal mixture comprising $N=N_{\mathrm{A}}+N_{\mathrm{B}}$ Brownian colloidal particles in $d=2$ spatial dimensions. The particles are in an area $S$ with a fixed total number density of $\rho=\frac{N}{S}$. Half of them are particles of type A; the other half are of type $\mathrm{B}$, with partial number density $\rho_{\mathrm{A}}=\rho_{\mathrm{B}}=\frac{\rho}{2}$. The colloidal suspension is held at fixed temperature $T$ via the bath of microscopic solvent particles. The colloidal particles of species $\mathrm{a}$ and $\mathrm{b}(\mathrm{a}, \mathrm{b} \in\{\mathrm{A}, \mathrm{B}\})$ are interacting via an effective Yukawa pair potential

$$
\frac{V_{\mathrm{ab}}(r)}{k_{\mathrm{B}} T}=U_{0} \sigma_{\mathrm{ab}} \frac{\exp \left[-\kappa\left(r-\sigma_{\mathrm{ab}}\right) / \sigma\right]}{r} .
$$

Here $r$ is the centre-to-centre separation, $k_{\mathrm{B}} T$ is the thermal energy, $U_{0}$ is a dimensionless amplitude, $\sigma$ is the particle diameter as a length scale and $\kappa$ is the inverse screening length. The set of diameters $\sigma_{\mathrm{ab}}$ is taken as

$$
\begin{aligned}
& \sigma_{\mathrm{AA}}=\sigma_{\mathrm{BB}}=\sigma, \\
& \sigma_{\mathrm{AB}}=\sigma(1+\Delta) .
\end{aligned}
$$

We chose $\Delta>0$ corresponding to positive non-additivity. This implies that the crossinteraction $V_{\mathrm{AB}}(r)$ is more repulsive than $V_{\mathrm{AA}}(r)=V_{\mathrm{BB}}(r)$, which drives phase separation into an A-rich and a B-rich phase.

The dynamics of the colloidal particles is overdamped Brownian motion. The friction constant $\xi=3 \pi \eta \sigma$ (with $\eta$ denoting the shear viscosity of the solvent) is assumed to be the same for both A and B particles. The constant external force for the $i$ th particle of species $a$, $\vec{F}_{i}^{(\text {a) }}$, acts differently on the two constituents of the binary mixture. It is $\vec{F}_{i}^{(\mathrm{A})}=\vec{e}_{z} F$ for A particles and $\vec{F}_{i}^{(\mathrm{B})}=-\vec{e}_{z} F$ for B particles. The stochastic Langevin equations for the colloidal trajectories $\vec{r}_{i}^{(\mathrm{a})}(t)$ with $i=1, \ldots, N_{\mathrm{a}}(\mathrm{a} \in\{\mathrm{A}, \mathrm{B}\})$ read

$$
\xi \frac{\mathrm{d} \vec{r}_{i}^{(\mathrm{a})}}{\mathrm{d} t}=\sum_{\substack{j=1 \\ j \neq i}}^{N_{\mathrm{a}}} \vec{F}_{i j}^{(\mathrm{aa})}+\sum_{j=1}^{N_{\mathrm{b}}} \vec{F}_{i j}^{(\mathrm{ab})}+\vec{F}_{i}^{(\mathrm{a})}+\vec{F}_{i}^{(\mathrm{R})}(t),
$$

where

$$
\vec{F}_{i j}^{(\mathrm{ab})}=-\vec{\nabla}_{\vec{r}_{i}^{(\mathrm{a})}} V_{\mathrm{ab}}\left(r_{i j}^{(\mathrm{ab})}\right),
$$

$r_{i j}^{(\mathrm{ab})}=\left|\vec{r}_{i}^{(\mathrm{a})}-\vec{r}_{j}^{(\mathrm{b})}\right|$ and $\mathrm{b}$ is the index complementary to $\mathrm{a}(\mathrm{b}=\mathrm{A}$ if $\mathrm{a}=\mathrm{B}$ and $\mathrm{b}=\mathrm{B}$ if $\mathrm{a}=\mathrm{A}$ ). The right-hand side includes all forces acting on the colloidal particles, namely the force resulting from interparticle interactions, the external constant force and the random forces $\vec{F}_{i}^{(\mathrm{R})}$ describing the collisions of the solvent molecules with the $i$ th colloidal particle. The latter are Gaussian random numbers with zero mean, ${\overrightarrow{F_{i}^{(R)}}}_{i}=0$, and variance

$$
\overline{\left(\vec{F}_{i}^{(\mathrm{R})}\right)_{\alpha}(t)\left(\vec{F}_{j}^{(\mathrm{R})}\right)_{\beta}\left(t^{\prime}\right)}=2 k_{\mathrm{B}} T \xi \delta_{\alpha \beta} \delta_{i j} \delta\left(t-t^{\prime}\right) .
$$

The subscripts $\alpha$ and $\beta$ stand for the two Cartesian components. In the absence of an external field and for $\Delta=0$, the model reduces to a two-dimensional Brownian Yukawa 
fluid in equilibrium which has been extensively investigated as far as structural and dynamical equilibrium correlations and freezing transitions are concerned [29-31]. For a positive $\Delta$ and vanishing external drive, our system will lead to equilibrium fluid-fluid phase separation including a critical point which has been studied in non-additive hard core models by means of theory and simulation; see e.g. [32-37].

We emphasize that our dynamics is overdamped motion and there is no inertia. The fluid is certainly different to a Newtonian one, since there is a quiescent solvent damping the particle's motion. We solve the Langevin equations of motion by means of Brownian dynamics simulations (BD) [38-40] using a finite time step $\Delta t$ and the technique of Ermak [41, 42]. The typical size of the time step was $0.003 \tau_{\mathrm{B}}$, where $\tau_{\mathrm{B}}=\xi \sigma^{2} / V_{0}$ is a suitable Brownian timescale. As a reference, the field-free case $\vec{F}=0$ is studied extensively first. This is an equilibrium situation. In the simulation set-up here, we put $N_{\mathrm{A}}=1000 \mathrm{~A}$ and $N_{\mathrm{B}}=1000 \mathrm{~B}$ particles into a rectangular cell of length $L$ in the $x$-direction and $D$ in the $z$-direction with $\frac{D}{L}=\frac{8}{5}$, such that the total colloidal number density is $\rho=\frac{N}{D L}$. Then the system spontaneously exhibits a fluid-fluid interface along the $x$-direction separating an A-rich from a B-rich fluid provided that the density is larger than the critical density. To have a single-interface system, without disturbing effects due to external walls, periodic boundary conditions are used in the $x$-direction while antiperiodic boundary conditions are used in the $z$-direction. With antiperiodic boundary conditions, the particle type is changed from A to B if an A particle is crossing a boundary in the $z$-direction and vice versa. In a finite slab around this boundary, all interactions are set to be equal, i.e. the cross-interaction is $V_{\mathrm{AB}}=V_{\mathrm{AA}}=V_{\mathrm{BB}}$. This avoids a second interface and minimizes finite size effects. Similar boundary conditions have been employed to study interfaces of symmetric polymer mixtures by Müller, Binder and co-workers [43].

We equilibrated $3 \times 10^{5}$ time steps corresponding to an equilibration time of $900 \tau_{\mathrm{B}}$ and gathered statistics again for a further simulation time of $900 \tau_{\mathrm{B}}$. We also checked that our data do not suffer from finite size effects by choosing a larger system with $N_{\mathrm{A}}=4000 \mathrm{~A}$ and $N_{\mathrm{B}}=4000 \mathrm{~B}$ particles with $\frac{D}{L}=\frac{3}{5}$. The antiperiodic boundary conditions force the interface to be parallel to the $x$-axis. All our investigations in non-equilibrium are with this larger system size.

With an equilibrated interface as a starting configuration we suddenly turn on the external field which drives the particles against the interface. An interface instability was observed. 300 different equilibrated starting configuration were then typically used in order to obtain time-dependent averages. For our non-equilibrium simulations, the antiperiodic boundary conditions in the $z$-direction have no consequences once the particles are driven against the interface.

\section{Density profiles and equilibrium fluid-fluid interfacial free energy}

In equilibrium, a phase-separated binary fluid mixture is characterized by its $z$-dependent partial density profiles $\rho_{\mathrm{A}}(z)$ and $\rho_{\mathrm{B}}(z)$ as defined via

$$
\rho_{\mathrm{a}}(z)=\left\langle\sum_{i=1}^{N_{\mathrm{a}}} \delta\left(z-z_{i}^{(\mathrm{a})}\right)\right\rangle
$$

where $\mathrm{a} \in\{\mathrm{A}, \mathrm{B}\}$ and $\langle\cdots\rangle$ denotes a canonical average. Furthermore, the associated fluidfluid interfacial free energy $\gamma$ is a key quantity. Since our model is two-dimensional, this interfacial free energy $\gamma$ corresponds to a line tension. In a symmetric binary mixture, the partial density profiles and the line tension $\gamma$ depend on the temperature and the total number density $\rho$. 

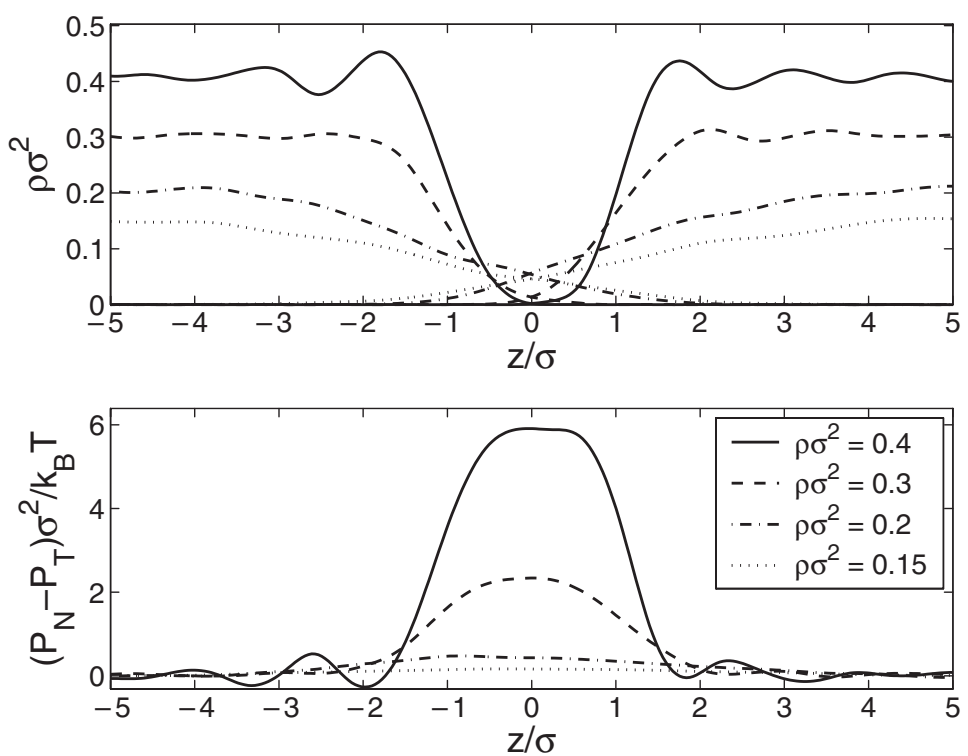

Figure 1. Averaged density profiles $\rho_{\mathrm{A}}(z)$ and $\rho_{\mathrm{B}}(z)$ of the two particle species and the anisotropy $P_{N}-P_{T}$ of the pressure tensor as a function of the distance $z$ perpendicular to the interface for $\Delta=1.6$ and for four different densities: $\rho \sigma^{2}=0.4$ (solid curves), $\rho \sigma^{2}=0.3$ (dashed curves), $\rho \sigma^{2}=0.2$ (dot-dashed curve) and $\rho \sigma^{2}=0.15$ (short dashed curves).

Obviously, due to the A-B symmetry of our model, the density profiles are symmetric, i.e. $\rho_{\mathrm{A}}(z)=\rho_{\mathrm{B}}(-z)$, if the interface position is at $z=0$. Computer simulation results for the density profiles for a strong positive non-additivity $\Delta=1.6$ and various bulk densities at a fixed temperature are presented in figure 1. For high densities one clearly identifies a depletion zone in the interface as generated by the large non-additivity. For densities closer to the critical one, however, the density profiles get flatter and particles interpenetrate mutually. For large bulk densities, a slight density oscillation shows up, as is typical for fluid interfaces when the Fisher-Widom line is exceeded [44]. Clearly, for the parameters chosen, the mixture is almost completely phase separated, i.e. the partial density of the A particles is practically zero in the $\mathrm{B}$ region and vice versa. Again this is different very close to the critical point.

We have also calculated the equilibrium line tension as a function of density for fixed temperature by using 'exact' computer simulation techniques. The interfacial free energy can be obtained by integration of the anisotropy of the pressure tensor [45-49]. In two spatial dimensions, this expression reads

$$
\gamma=\int_{-\infty}^{\infty}\left[P_{N}(z)-P_{T}(z)\right] \mathrm{d} z
$$

where $P_{N}(z)=p_{z z}(z)$ and $P_{T}(z)=p_{x x}(z)$ are the diagonal components of the local pressure tensor. This local pressure tensor has the following form [50-54]:

$$
\begin{aligned}
p_{\alpha \beta}(z)=\left(\rho_{\mathrm{A}}(z)+\rho_{\mathrm{B}}(z)\right) k_{\mathrm{B}} T \delta_{\alpha \beta} \\
\quad-\frac{1}{L} \sum_{\substack{1 \leqslant i<j \leqslant N_{\mathrm{a}} \\
\mathrm{a}=\mathrm{A}, \mathrm{B}}}\left(\vec{r}_{i j}^{(\mathrm{aa})}\right)_{\alpha}\left(\vec{F}_{i j}^{(\mathrm{aa})}\right)_{\beta} \frac{1}{\left|z_{i j}^{(\mathrm{aa})}\right|} \Theta\left(\frac{z-z_{i}^{(\mathrm{a})}}{z_{i j}^{(\mathrm{aa})}}\right) \Theta\left(\frac{z_{j}^{(\mathrm{a})}-z}{z_{i j}^{(\mathrm{aa})}}\right)
\end{aligned}
$$



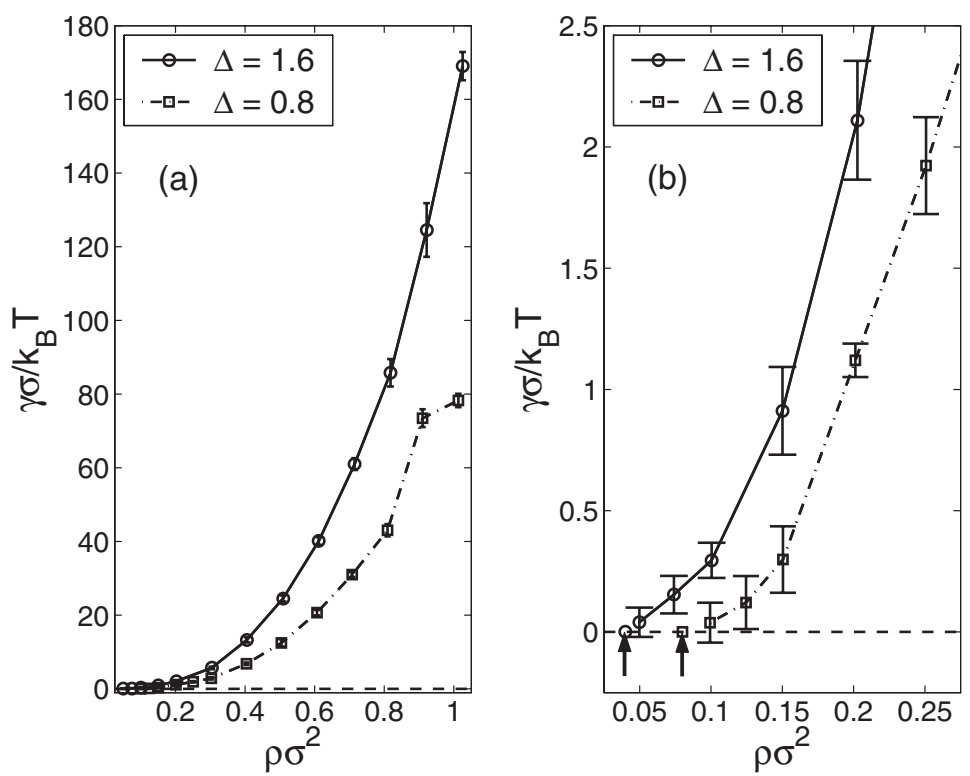

Figure 2. The reduced line tension $\gamma \sigma / k_{\mathrm{B}} T$ as a function of the reduced bulk density $\rho \sigma^{2}$ for two different non-additivities $\Delta=0.8$ (solid curve) and $\Delta=1.6$ (dot-dashed curve). (a) Away from the critical point. (b) Closer to the critical point. The estimates for the critical density are shown as arrows.

$\left.+\sum_{\substack{1 \leqslant i \leqslant N_{\mathrm{A}} \\ 1 \leqslant j \leqslant N_{\mathrm{B}}}}\left(\vec{r}_{i j}^{(\mathrm{AB})}\right)_{\alpha}\left(\vec{F}_{i j}^{(\mathrm{AB})}\right)_{\beta} \frac{1}{\left|z_{i j}^{(\mathrm{AB})}\right|} \Theta\left(\frac{z-z_{i}^{(\mathrm{A})}}{z_{i j}^{(\mathrm{AB})}}\right) \Theta\left(\frac{z_{j}^{(\mathrm{B})}-z}{z_{i j}^{(\mathrm{AB})}}\right)\right\rangle$

where the subscripts $\alpha$ and $\beta$ stand for the two Cartesian components, $\vec{r}_{i j}^{(\mathrm{ab})}=\vec{r}_{i}^{(\mathrm{a})}-\vec{r}_{j}^{(\mathrm{b})}$, $z_{i j}^{(\mathrm{ab})}=z_{i}^{(\mathrm{a})}-z_{j}^{(\mathrm{b})}$ and $\Theta(z)$ is the Heaviside step function. The $z$ dependence of the anisotropy of the pressure tensor is plotted in figure 1 as well. As can be deduced from figure 1, its main weight is centred in the interface position around $z=0$. The oscillations of the density field for high densities correlate to that of the pressure tensor.

Simulation results for the density-dependent line tension $\gamma(\rho)$ for two different nonadditivities $\Delta=0.8$ and 1.6 are presented in figure 2. Most of our calculations were for parameter combinations well away from the critical point where the line tension is mainly governed by internal energy, such that the anisotropy of the pressure tensor is significant, resulting in a relative small statistical error for $\gamma$; see figure 2(a). Some further points are also for smaller densities closer to the critical point; see figure 2(b). By crudely extrapolating the data one could estimate the critical density to be at $\rho_{\mathrm{c}} \sigma^{2} \approx 0.08$ for $\Delta=0.8$ and $\rho_{\mathrm{c}} \sigma^{2} \approx 0.04$ for $\Delta=1.6$ as indicated by arrows in figure 2(b). This extrapolation was cross-checked by mapping our Yukawa system onto that of effective non-additive hard discs using the prescription of Barker and Henderson for the effective hard core diameter [55]. The critical point can then be read off from that of a non-additive symmetric hard disc binary mixture which has been studied in detail via theory and simulation by Giaquinta and co-workers [36]. We find good agreement with our extrapolation as compared to the mapping procedure. As can be further deduced from figure 2, the line tension $\gamma(\rho)$ is strongly increasing with density. This is expected, as a density increase means a smaller spacing between A and B particles, such that the energetic non-additivity gets more pronounced. At fixed density and temperature, the line 
tension increases with $\Delta$, which is clearly due to the fact that a larger non-additivity leads to a larger energy cost of different particle species meeting at the interface.

\section{Classical Rayleigh-Taylor instability}

The classical Rayleigh-Taylor instability is obtained for a heavy incompressible liquid on top of a lighter incompressible liquid $[1,56]$. A small harmonic interfacial undulation with a wavelength $\lambda$ yields a favourable decrease in potential energy but at the same time a free energy penalty due to the increasing arc length of the interface which costs line tension. If the wavelength is larger than a critical value, $\lambda>\lambda_{\mathrm{c}}$, however, the penalty is smaller than the gain, such that an unstable mode whose amplitude grows in time is present. The critical wavelength can be calculated in our two-dimensional situation as

$$
\lambda_{\mathrm{c}}=\frac{2 \pi}{k_{\mathrm{c}}}=2 \pi \sqrt{\frac{\gamma}{\left|\vec{F}^{(\mathrm{A})}-\vec{F}^{(\mathrm{B})}\right| \rho}}
$$

where $\gamma$ is the line tension as introduced in the previous section. Since the concept of line tension is a macroscopic one, use of equation (10) is only justified as long as $\lambda_{\mathrm{c}}$ is much larger than any microscopic distance, e.g. the mean interparticle spacing $a=\frac{1}{\sqrt{\rho}}$. This requires that the line tension has to be much larger than the driving force difference:

$$
\lambda_{\mathrm{c}} \gg a \quad \Rightarrow \quad \gamma>\left|\vec{F}^{(\mathrm{A})}-\vec{F}^{(\mathrm{B})}\right| \text {. }
$$

In the opposite limit

$$
\gamma \ll\left|\vec{F}^{(\mathrm{A})}-\vec{F}^{(\mathrm{B})}\right|
$$

it is expected that the classical concept of the Rayleigh-Taylor instability will break down. We shall explore this case in detail in section 6 . Another question concerns the applicability of the Rayleigh-Taylor instability criterion when $\lambda_{\mathrm{c}}$ is of a similar order to the mean interparticle spacing $a$. As we shall show below, the criterion works remarkably well even if $\lambda_{\mathrm{c}}$ is close to $a$.

\section{Results for high and moderate interfacial tensions}

Typical snapshots of our non-equilibrium computer simulations are presented in figure 3 for four different times and for two different densities $\rho^{*}=\rho \sigma^{2}=0.2,0.4$ and two different strengths of the driving force $F^{*}=F \sigma / k_{\mathrm{B}} T=10,40$. The parameter combinations investigated and the corresponding ratios of the line tension $\gamma$ and the driving force $F$ are summarized in table 1. They are of the order of 1 (for combination A-C) or a bit smaller (for combination D), such that the Rayleigh-Taylor instability wavelength is larger than or of the order of the mean interparticle spacing $a$; see again table 1 for the ratio $\lambda_{c} / a$. We call an interface tension 'high' if $\lambda_{\mathrm{c}} / a>1$ and 'intermediate' if $\lambda_{\mathrm{c}} / a \approx 1$. This is in contrast to the case for low surface tensions (combination E) where $\lambda_{c} / a$ is significantly smaller than 1 ; this is studied in section 6 .

The system was started with an equilibrated single-interface situation. The initial interface is fairly smooth but carries capillary fluctuations. The density depletion shown in figure 1 manifests itself as a clear void region in the interface, reminiscent of a 'forest-aisle' which is induced by the strong energy cost when two different particle species meet.

Then instantaneously the external driving forces were turned on, forcing the particles to drift against each other. This first causes a local density increase close to the interface. Then interface undulations become more pronounced, deforming the flat interface. This is the most efficient channel for different particle species to reverse their height. A typical characteristic undulation wavelength can be identified from the simulation snapshot, which we shall 

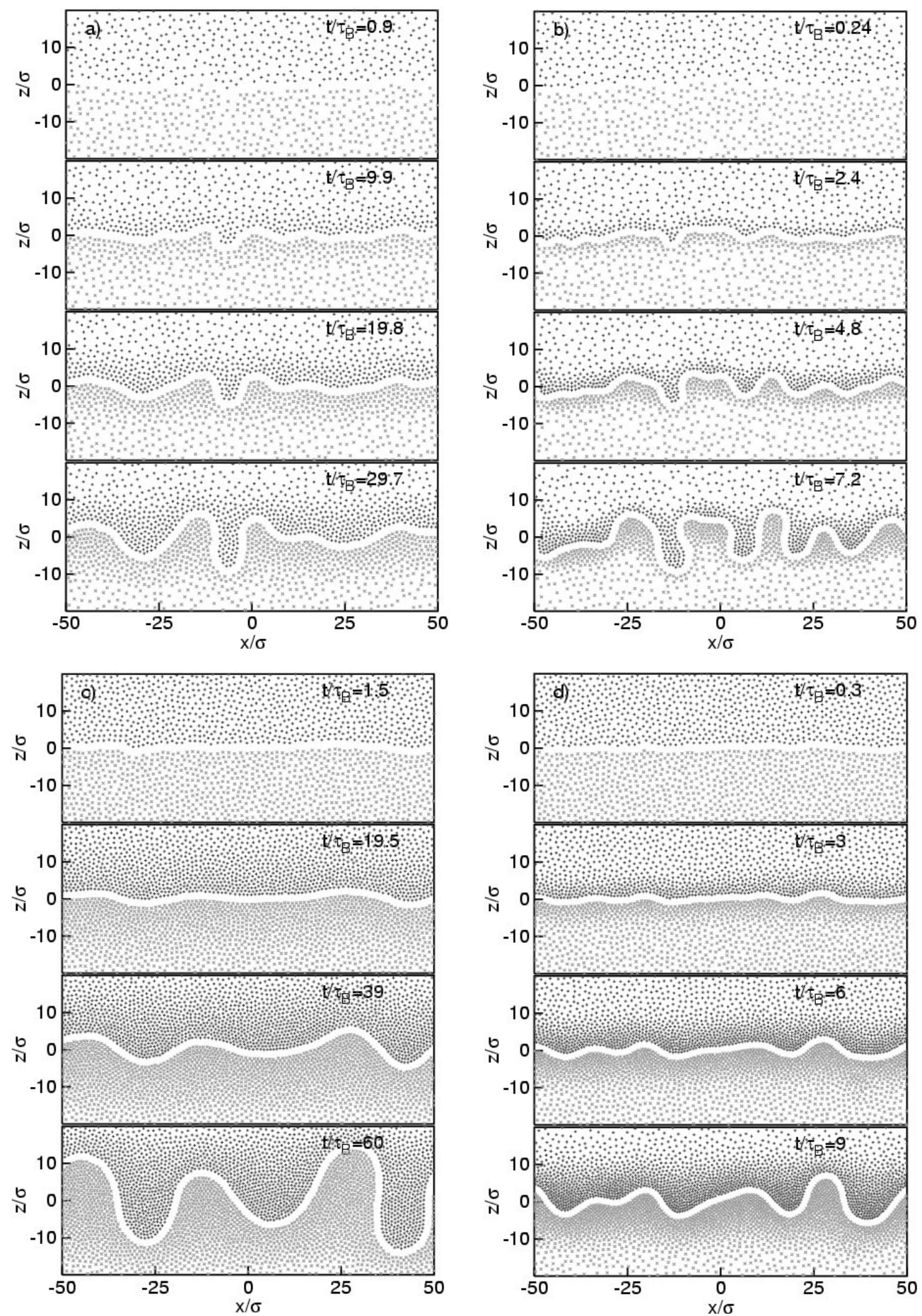

Figure 3. Typical simulation snapshots for an interface in non-equilibrium with an external drive. There is a sharp interface between an A-rich and a B-rich fluid phase. The starting configuration was an equilibrated interface at $z=0$. Four different times are shown as given in the legend. (a) $\rho \sigma^{2}=0.2$ and $F^{*}=F \sigma / k_{\mathrm{B}} T=10$, (b) $\rho \sigma^{2}=0.2$ and $F^{*}=F \sigma / k_{\mathrm{B}} T=40$, (c) $\rho \sigma^{2}=0.4$ and $F^{*}=F \sigma / k_{\mathrm{B}} T=10$, (d) $\rho \sigma^{2}=0.4$ and $F^{*}=F \sigma / k_{\mathrm{B}} T=40$. 
Table 1. Summary of the five parameter combinations studied in the paper. The corresponding ratios $\gamma / F$ and $\lambda_{\mathrm{c}} / a$ are also given.

\begin{tabular}{lllll}
\hline & $\rho^{*}$ & $F^{*}$ & $\gamma / F$ & $\lambda_{\mathrm{c}} / a$ \\
\hline $\mathrm{A}$ & 0.4 & 10 & 1.3 & 5 \\
$\mathrm{~B}$ & 0.4 & 40 & 0.32 & 2.5 \\
$\mathrm{C}$ & 0.2 & 10 & 0.2 & 2 \\
$\mathrm{D}$ & 0.2 & 40 & 0.05 & 1 \\
$\mathrm{E}$ & 0.075 & 80 & 0.002 & 0.2 \\
\hline
\end{tabular}

quantify later. Furthermore, nonlinear effects such as interfacial overhangs ('mushrooms') can be seen (e.g. in figure 3(a) for the largest time) until the mixtures penetrate through each other and the height reversal is complete.

In order to quantify the structural signatures of the interface instability further, we consider the Fourier transform of the interface position as a function of time. In detail, let $h(x, t)$ be the interface position of a given configuration at a time $t$, and

$$
\tilde{h}(k, t)=\int_{0}^{L} h(x, t) \exp (-\mathrm{i} k x) \mathrm{d} x
$$

be the corresponding Fourier transform. We are interested in the time dependence of the averaged power spectral density which is defined as

$$
P(k, t)=\left\langle\tilde{h}(k, t) \tilde{h}^{*}(k, t)\right\rangle
$$

where $\langle\cdots\rangle$ now denotes an ensemble average over the initial equilibrated configurations which dynamically evolved after a time $t$. If, at a given $t, P(k, t)$ possesses a sharp maximum at $k=k_{\mathrm{m}}$, this implies that the interface will exhibit mainly undulations with a wavelength of $\lambda_{\mathrm{m}}=2 \pi / k_{\mathrm{m}}$.

As an aside, let us remark that an alternative way of obtaining the line tension $\gamma$ is via the equilibrium capillary wave spectrum $P(k, t=0)$ which behaves as

$$
P(k, t=0)=\frac{k_{\mathrm{B}} T}{\gamma} \frac{L}{k^{2}}
$$

for small wavevectors $k[53,57,58]$. We have checked that the values for the line tension $\gamma$ as obtained from this formula are consistent with those obtained from equation (8).

We are now in a position to define a differential growth rate $\Gamma(k, t)$ via

$$
\Gamma(k, t)=\frac{1}{2} \frac{1}{P(k, t)} \frac{\mathrm{d}}{\mathrm{d} t} P(k, t) .
$$

A positive sign of $\Gamma(k, t)$ implies that a mode of wavenumber $k$ is growing at a time $t$ while a negative sign means that—at a given time $t$ - the mode is decreasing.

In detail, the following numerical procedure was used to obtain the actual interface position $h(x, t)$ : for a given particle configuration at time $t$, we construct Voronoi cells around each particle and define the interface on the basis of the associated Voronoi polygons. The polygon vertices that belong to cells of particles of both species A and B define a set of $M^{*}$ nonequidistant coordinates $\left(h_{n}, x_{n}\right)_{n=1, \ldots, M^{*}}$. Now we eliminate the protuberance in the interface profile to get a functional relation for $h(x)$. For further analysis we interpolate $\left(h_{n}, x_{n}\right)_{n=1, \ldots, M^{*}}$ using a cubic spline and eliminate sharp bends by using a simple low pass filter:

$$
h_{n} \rightarrow h_{n}^{*}=\left(h_{n}+h_{n+1}\right) / 2
$$

where $n=1, \ldots, M$ and $h_{M+1} \equiv h_{1}$. This procedure results in a unique $z$ position $h(x, t)$ of the interface at a given time $t$. 

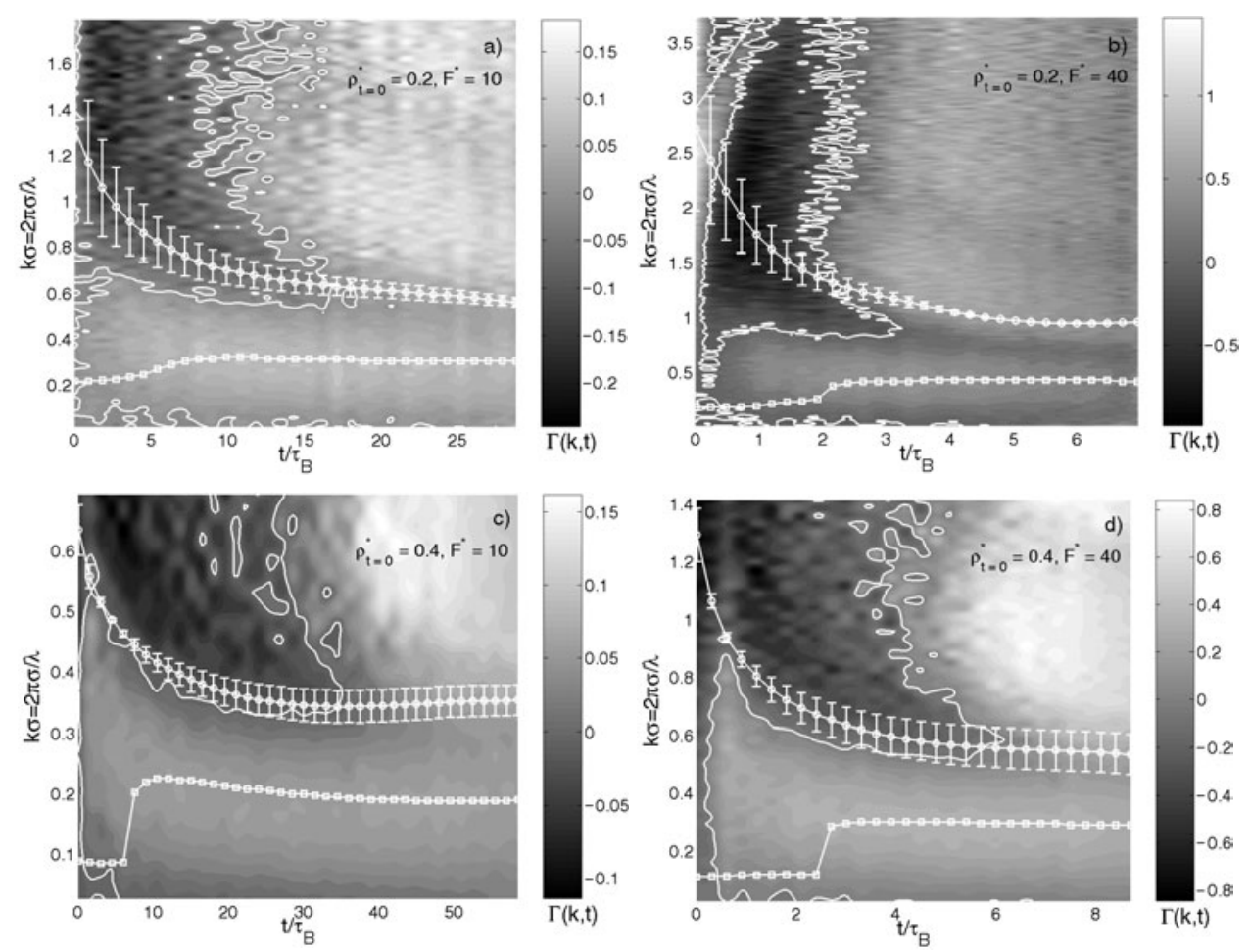

Figure 4. Contour plots of the growth rate $\Gamma(k, t)$. Zero growth is shown by the white line. The rescaled classical Rayleigh-Taylor wavelength $2 \pi / \lambda_{\mathrm{c}}$ is shown by the circles. Here a timedependent maximum $\rho_{\mathrm{m}}$ of the total density at the interface is used. The statistical error stems from the uncertainty of this density. The position of the second maximum in the height-height correlation function is shown by the squares. The corresponding microscopic wavenumber as defined by $2 \pi \rho_{\mathrm{m}}^{1 / 2}$ and gives an estimate for the threshold where a microscopic length scale is realized. (a) $\rho \sigma^{2}=0.2$ and $F^{*}=F \sigma / k_{\mathrm{B}} T=10$, (b) $\rho \sigma^{2}=0.2$ and $F^{*}=F \sigma / k_{\mathrm{B}} T=40$, (c) $\rho \sigma^{2}=0.4$ and $F^{*}=F \sigma / k_{\mathrm{B}} T=10$, (d) $\rho \sigma^{2}=0.4$ and $F^{*}=F \sigma / k_{\mathrm{B}} T=40$.

Results for the differential growth rate $\Gamma(k, t)$ are shown in figure 4 as a contour plot in the plane spanned by the time $t$ and the wavevector $k$. As in figure 3, data for the four different parameter combinations A-D are presented. The white zero line summarizes points where $\Gamma(k, t)$ vanishes and separates two regimes with growing (stable) modes and damped (unstable) wavenumbers. Although this line is noisy, it can be read off from figure 4 that there are a whole band of wavenumbers which are growing after an induction time of roughly $\tau_{\mathrm{B}}$. The lower limit of unstable wavenumbers is significantly larger than the inverse simulation box length, while its upper limit is always smaller than a microscopic wavenumber of the order of $2 \pi / a$. Between these two boundaries, roughly at the arithmetic mean of the two limits, there is a wavenumber with a maximal growth rate. The first basic observation is that for parameter combinations $\mathrm{A}-\mathrm{C}$ where $\lambda_{\mathrm{c}} / a$ is larger than 1 , the upper limit of unstable wavenumbers is decreasing with time. This has to do with our equilibrated starting configuration. When the external field is turned on, there is a sedimentation-like process towards the interface which yields a local density increase at the interface. This behaviour can be directly seen in the simulation snapshots of figure 3. Thereby the interface is getting stiffer as a function of time. We call this important effect self-healing of the interface; i.e. as a function of time, the interface is getting less vulnerable with respect to short wavelength undulations. A possible destruction 
of the interface is efficiently blocked by a density accumulation. It is tempting to correlate this with an effective interface tension with a scaled density as obtained e.g. for the maximal total density $\rho_{\mathrm{m}}$ close to the interface. This maximal density $\rho_{\mathrm{m}}$ is a function of time. If one plugs this time-dependent density into the expression for $\gamma(\rho)$, one obtains a dynamically rescaled Rayleigh-Taylor expression (10). The corresponding wavenumber is also shown in figure 4; see the open circles with error bars. Clearly the basic effect is encaptured by the dynamical rescaling, as the classical Rayleigh-Taylor unstable wavenumber coincides well with the upper unstable limit. The agreement is encouraging in particular for parameter combinations A-C where $\lambda_{\mathrm{c}} / a>1$.

A special remark is in order as regards parameter combination D where $\lambda_{\mathrm{c}}$ is of the order of $a$. In fact, we estimate a microscopic wavenumber by $2 \pi \sqrt{\rho_{\mathrm{m}}}$ and data for this wavevector $2 \pi \sqrt{\rho_{\mathrm{m}}}$ are included in figure 4 as crosses. Even in this case, the comparison between the upper unstable wavenumber and the scaled Rayleigh-Taylor prediction is qualitative. This implies that the classic Rayleigh-Taylor criterion is astonishingly robust even close to molecular spacings.

On the other hand, the maximal growth rate is seen as a light region for small wavevectors. This wavenumber is slightly decreasing with time as well. We have further compared the wavenumber of maximal growth by calculating the second maximum of the height-height correlation function which is defined as

$$
C(k, t)=\left\langle h(x, t) h\left(x+\frac{2 \pi}{k}, t\right)\right\rangle .
$$

The position of the second maximum of $C(k, t)$ is also included as a square line in figure 4 . It is increasing as a function of time towards larger wavenumbers, i.e. smaller wavelengths. The increase is small and confined to a small 'induction time' of the process. We attribute this to an initial process, which has to do with the fact that due to the non-homogeneous capillary wave spectrum equation (15), undulations with larger wavelengths have initially a larger amplitude and have therefore more weight in $C(k, t)$. This gives rise to the crossover to higher wavenumber after an induction time, which is fairly sharp for the parameter combinations $\mathrm{A}$ and $\mathrm{B}$. We further note that at higher $k$ the growth rate is becoming positive for larger times. This is due to the fact that the interface position is no longer sinusoidal, but starts to exhibit sharp parts close to overhangs. This is turn will generate higher Fourier modes that grow and this is what is indicated by the zero line at higher $k$ and larger times. Moreover the zero line at high $k$ and smaller times (in figures 4(a) and (b)) is statistical noise. Since we are dealing with a compressible Brownian fluid in a quiescent solvent, the standard analysis of the growth rates [1] for an incompressible Newtonian fluid does not apply. In fact, if one takes the traditional formula with the associated viscosities of the Brownian fluids (which we calculated following the approach of Heyes et al [59]), the results differ by more than an order of magnitude. An appropriate theoretical description for our set-up is based on dynamical density functional theory, as recently developed in [27], but this highly nontrivial analysis is beyond the scope of the present paper.

Further results for the time-resolved penetration heights $H$ are presented in figure 5. We see clearly two regimes: for small times, there is an exponential growth law (see the fitted exponential curve in figure 5) whose growth rate compares well with our numerical data for the maximal growth rate in figure 4(a). For larger times, this crosses over to a linear growth law as expected for an overdamped fluid. Here a linear function fits the data extremely well with a slope 0.51 that is smaller than $F / \xi=2 / 3 \approx 0.67$, which is the slope for a single driven particle in the quiescent solvent for our parameter combinations $\left(F^{*}=10, \rho^{*}=0.2\right.$ and $U_{0}=15$ ). This shows the qualitative difference from molecular fluids governed by molecular dynamics where there is a quadratic growth law of $H$ in time [60]. 

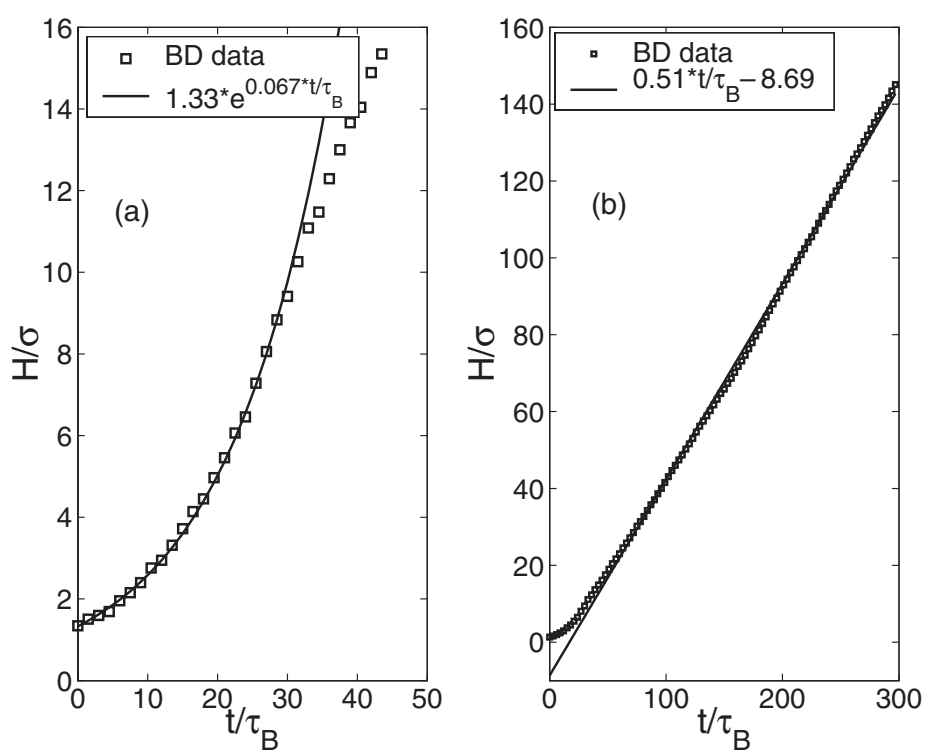

Figure 5. The reduced amplitude of the interface excursion (penetration height) $H / \sigma$ versus reduced time $t / \tau_{\mathrm{B}}$ for $\rho \sigma^{2}=0.2$ and $F^{*}=10$. (a) Simulation data (squares) as well as the exponential short time fit $1.33 \exp \left(0.067 t / \tau_{\mathrm{B}}\right)$ (solid curve). (b) Simulation data (squares) as well as the linear long time fit $0.51 t / \tau_{\mathrm{B}}-8.69$ (solid line).

In order to elucidate long time and finite size effects, we have performed a further simulation with $2 \times 10^{5}$ particles and a simulation time of $300 \tau_{\mathrm{B}}$. A corresponding snapshot is shown in figure 6 . Although there are complex substructures within the penetration regions of the two interfaces, the interface stays fairly smooth even for long times, again in contrast to Newtonian fluids.

\section{Results for low interfacial tensions}

Computer simulations similar to those in the previous section were carried out, but now in the regime where the line tension is low in the sense of the criterion of equation (12), such that $\lambda_{\mathrm{c}}$ is significantly smaller than $a$. Simulation snapshots are shown for $\rho^{*}=0.075$ close to the estimated critical density in figure 7 . In this case, the interface can be penetrated by the strongly driven particles and $\gamma / F=0.002$ and $\lambda_{\mathrm{c}} / a=0.2$; see the parameter combination $\mathrm{E}$ in table 1.

In penetrating the interface, the particles form lanes; this is similar to the behaviour in additive mixtures in $[25,26]$. The width of the lanes is comparable to the correlation length of the mixture, meaning that single worms of particles are formed [27]. At the head of the lanes both spikes and extrusions are visible. A similar behaviour is found for even smaller densities below the critical density where the system is mixed in equilibrium. Hence the RayleighTaylor criterion would predict a submolecular unstable wavelength, but the actual realized instability wavelength is a molecular correlation length of the system of the order of $a$.

\section{Conclusions}

In conclusion, we have studied the onset of the Rayleigh-Taylor interface instability on length scales of interparticle distances for a compressible Brownian fluid mixture. Depending on 


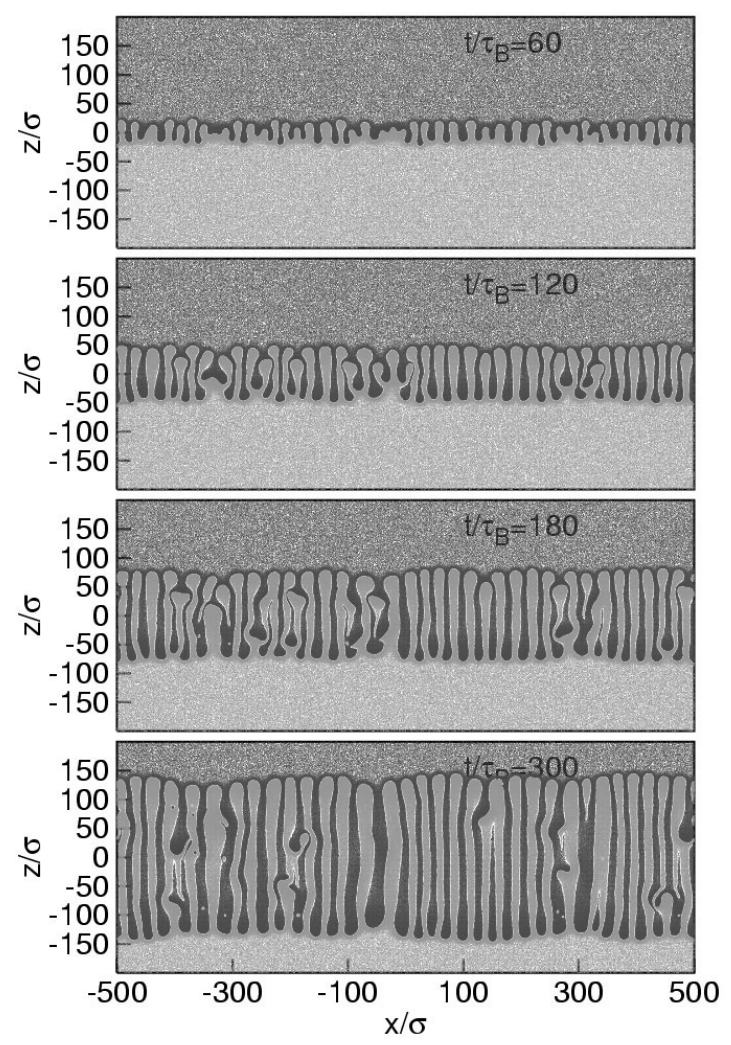

Figure 6. Simulation snapshots for a larger system size containing $2 \times 10^{5}$ particles up to a simulation time of $300 \tau_{\mathrm{B}}$ for $\rho \sigma^{2}=0.2$ and $F^{*}=10$. Four different times are shown, as given in the legend.

the equilibrium interfacial tension, either a direct transition towards lane formation or the macroscopic Rayleigh-Taylor instability was found. An interesting dynamical effect is the self-healing mechanism for the interface which is produced by a density accumulation of particles driven against each other, which then causes an increase of the instability wavelength as a function of time.

It would be interesting to study the dynamical process of phase separation by starting from a completely mixed system under the influence of the driving forces. Here one would expect a subtle interplay between phase separation kinetics which typically results in fractals and lane formation [61]. One should further investigate more extensively the dependence on the 'sedimentation height', i.e. how the data are affected by a larger initial $D$ in the $z$-direction perpendicular to the interface. Moreover. it would be interesting to start the simulation by impressing a prescribed wavelength as an interface undulation and check its differential growth. A third set-up for another initial configuration which is typically used for the molecular dynamics simulations [10] would be to equilibrate first with respect to the external field with a fixed interface, as induced e.g. by a thin hard platelet separating the two fluid phases. The effects of different starting configurations on the onset of the Rayleigh-Taylor instability will pose another challenging problem.

For simplicity, all our simulations were done in two spatial dimensions. In three dimensions, similar effects should persist. However, the characteristic wavelength now has 


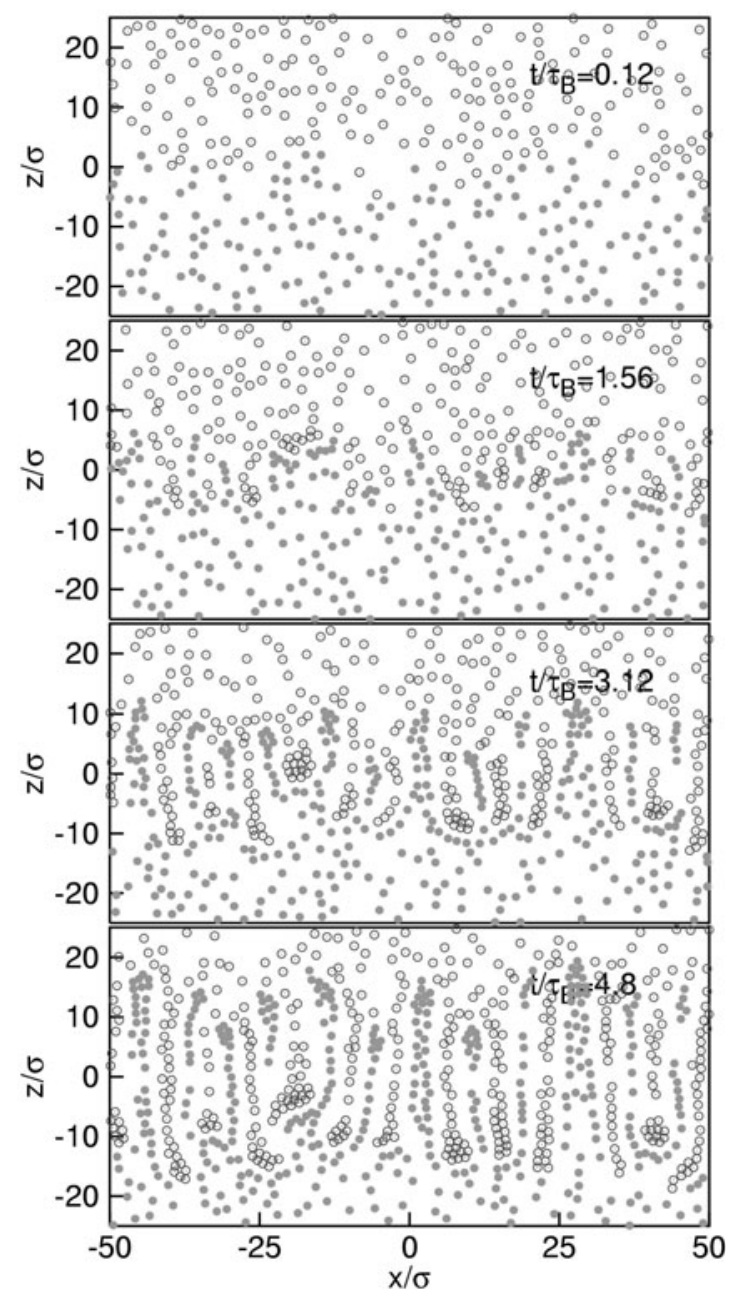

Figure 7. A typical simulation snapshot for an interface in non-equilibrium with an external drive. There is a smooth interface between an A-rich and a B-rich fluid phase. The starting configuration was an equilibrated interface at $z=0$. Four different times are shown as given in the legend. The parameters are $\rho \sigma^{2}=0.075$ and $F^{*}=F \sigma / k_{\mathrm{B}} T=80$.

two components parallel to the interface. The self-stabilizing effect of the interface and the two extreme limits should be similar to that in two dimensions. The lane formation, for instance, has also been shown to be present in three spatial dimensions.

Furthermore, we have neglected solvent-mediated hydrodynamic interactions between the colloids by assuming simple Brownian dynamics in our model. While this is justified for low colloid packing fraction and small driving forces, hydrodynamic interactions are expected to become relevant for large driving strengths and high packing fractions of colloids.

Let us finally discuss some possible experimental tests. Strongly non-additive mixtures which phase separate into two fluid phases are found in colloid/polymer mixtures which exhibit colloid-rich and colloid-poor fluid phases for a size ratio of about 0.5 or larger between the polymer and the colloid [62]. Recently a phase-separating mixture of colloids and polymers was observed in sedimentation, by Aarts and co-workers [17]. In particular, it was observed 
that large regions of phase-separated colloid-rich and colloid-poor phases exhibit a transition towards lane formation. The characteristic wavelength is of the order of that given by the Rayleigh-Taylor instability equation (10) [63]. A full quantitative comparison has still to be performed. Another system where such lane formation has been seen and which could be a candidate for providing a quantitative comparison is a xanthan-colloid mixture [64].

\section{Acknowledgments}

We thank D G A L Aarts, B J Alder, K Binder, R Blaak, J Dzubiella, G Morfill, K H Spatschek, K R Mecke and V Steinberg for helpful remarks. Financial support from the DFG (Sonderforschungsbereich TR6 subproject D3) is gratefully acknowledged.

\section{References}

[1] Chandrasekhar S 1961 Hydrodynamic and Hydromagnetic Stability (Oxford: Oxford University Press)

[2] Cross M C and Hohenberg P C 1993 Rev. Mod. Phys. 65851

[3] Ignes-Mullol J and Oswald P 2000 Phys. Rev. E 613969

[4] Durand I, Kassner K, Misbah C and Müller-Krumbhaar H 1998 Phys. Rev. E 586027

[5] Lindner A, Bonn D, Poire E C, Ben Amar M and Meunier J 2002 J. Fluid Mech. 469237

[6] Coussot P 1999 J. Fluid Mech. 380363

[7] Alda W, Dzwinel W, Kitowski J, Moscinski J, Pogoda M and Yuen D A 1998 Comput. Phys. 12595

[8] Pan T W, Joseph D D and Glowinski R 2001 J. Fluid Mech. 43423

[9] Dzwinel W and Yuen D A 2001 Int. J. Mod. Phys. C 1291

[10] Kadau K, Germann T C, Hadijconstantinou N G, Lomdahl P S, Dimonte G, Holian B L and Alder B J 2004 Proc. Natl. Acad. Sci. USA 1015851

[11] Murray C A and Van Winkle D H 1987 Phys. Rev. Lett. 581200

[12] Pusey P N 1991 Liquids, Freezing and the Glass Transition ed J P Hansen, D Levesque and J Zinn-Justin (Amsterdam: North-Holland)

[13] Löwen H 2001 J. Phys.: Condens. Matter 13 R415

[14] de Hoog E H A, Kegel W K, van Blaaderen A and Lekkerkerker H N W 2001 Phys. Rev. E 64021407

[15] Brader J M, Evans R, Schmidt M and Löwen H 2002 J. Phys.: Condens. Matter 14 L1

[16] Velikov K P, Christova C G, Dullens R P A and van Blaaderen A 2002 Science 296106

[17] Aarts D G A L, van der Wiel J H and Lekkerkerker H N W 2003 J. Phys.: Condens. Matter 15 S245

[18] Hoogenboom J P, van Langen-Suurling A K, Romijn H and van Blaaderen A 2003 Phys. Rev. Lett. 90138301

[19] Hoogenboom J P, Yethiraj A, van Langen-Suurling A K, Romijn J and van Blaaderen A 2002 Phys. Rev. Lett. 89256104

[20] Löwen H, Hansen J P and Roux J N 1991 Phys. Rev. A 441169

[21] Völtz C, Pesch W and Rehberg I 2001 Phys. Rev. E 65011404

[22] Völtz C 2003 Phys. Rev. E 68021408

[23] Reichhardt C, Olson-Reichhardt C J and Hastings M B 2004 Phys. Rev. E 69056115

[24] MacGowan D and Evans D J 1986 Phys. Rev. A 342133

[25] Dzubiella J, Hoffmann G P and Löwen H 2002 Phys. Rev. E 65021402

[26] Dzubiella J and Löwen H 2002 J. Phys.: Condens. Matter 149383

[27] Chakrabarti J, Dzubiella J and Löwen H 2003 Europhys. Lett. 61415

[28] Löwen H and Dzubiella J 2003 Faraday Discuss. 12399

[29] Löwen H 1992 J. Phys.: Condens. Matter 410105

[30] Löhle B and Klein R 1997 Physica A 235224

[31] Naidoo K J and Schnitker J 1994 J. Chem. Phys. 1003114

[32] Ehrenberg V, Schaink H M and Hoheisel C 1990 Physica A 169365

[33] Nielaba P 1996 Int. J. Thermophys. 17157

[34] Lomba E, Alvarez M, Lee L and Almarza N 1996 J. Chem. Phys. 1044180

[35] Al-Naafa M, El-Yakubu J B and Hamad E Z 1999 Fluid Phase Equilib. 15433

[36] Saija F and Giaquinta P V 2002 J. Chem. Phys. 1175780

[37] Góźdź W T 2003 J. Chem. Phys. 1193309

[38] Löwen H and Hoffmann G P 1999 Phys. Rev. E 603009 
[39] Hoffmann G P and Löwen H 2001 J. Phys.: Condens. Matter 139197

[40] Löwen H, Hansen J P and Roux J N 1991b Phys. Rev. A 441169

[41] Allen M P and Tildesley D J 1987 Computer Simulations of Liquids (Oxford: Oxford University Press)

[42] Ermak D L 1975 J. Chem. Phys. 624189

[43] Werner A, Schmid F, Müller M and Binder K 1997 J. Chem. Phys. 1078175

[44] Archer A J and Evans R 2001 Phys. Rev. E 64041501

[45] Kirkwood J G and Buff F P 1949 J. Chem. Phys. 17338

[46] Smit B 1988 Phys. Rev. A 373431

[47] Alejandre J, Tildesley D J and Chapela G A 1995 J. Chem. Phys. 1024574

[48] Pandey R B, Milchev A and Binder K 1997 Macromolecules 301194

[49] Milchev A and Binder K 2001 J. Chem. Phys. 1148610

[50] Irving J H and Kirkwood J G 1950 J. Chem. Phys. 18817

[51] Ono S and Kondo S 1960 Encyclopedia of Physics ed S Flügge (Berlin: Springer)

[52] Schofield P and Henderson J H 1982 Proc. R. Soc. A 379231

[53] Rowlinson J S and Widom B 1982 Molecular Theory of Capillarity (Oxford: Oxford University Press)

[54] Walton J P R B, Tildesley D J, Rowlinson J S and Henderson J R 1983 Mol. Phys. 481357

[55] Hansen J P and McDonald I R 1990 Theory of Simple Liquids 2nd edn (New York: Academic)

[56] Sharp D H 1984 Physica D 123

[57] Buff F P, Lovett R A and Stillinger F H 1965 Phys. Rev. Lett. 15621

[58] Weeks J D 1977 J. Chem. Phys. 673106

[59] Brańka A C and Heyes D M 1997 Phys. Rev. E 555713

[60] Dimonte G and Schneider M 2000 Phys. Fluids 12304

[61] Löwen H, Likos C N, Blaak R, Auer S, Froltsov V, Dzubiella J, Wysocki A and Harreis H M 2004 Colloidal Suspensions Driven by External Fields (AIP Conf. Proc. vol 708) ed M Tokuyama and I Oppenheim (New York: American Institute of Physics) pp 3-7 (ISBN 0-7354-0183-7)

[62] Dijkstra M, Brader J M and Evans R 1999 J. Phys.: Condens. Matter 1110079

[63] Aarts D G A L 2003 private communication

[64] Koenderink G H, Aarts D G A L, Villeneuve V W A, Philipse A P, Tuinier R and Lekkerkerker H N W 2003 Biomacromolecules 4129 